\title{
Ising Model on the Generalized Bruhat-Tits Tree
}

\author{
Yu. M. Zinoviev \\ Steklov Mathematical Institute, GSP-1, SU-117333 Moscow, USSR
}

\begin{abstract}
The partition function and the correlation functions of the Ising model on the generalized Bruhat-Tits tree are calculated. We computed also the averages of these correlation functions when the corresponding vertices are attached to the boundary of the generalized Bruhat-Tits tree.
\end{abstract}

\section{Introduction}

The Ising model on the Cayley tree turns out to be very interesting $[1,2]$. The Cayley tree $T$ is manifestly determined to be a connected infinite graph with no loops, each vertex of $T$ being connected with exactly $p+1$ nearest neighbour vertices by links. If $p$ is a prime number, the Cayley tree is called the Bruhat-Tits tree. The branch $B_{z}$ is defined to be a connected subtree with the only boundary vertex $z$ of the graph $T \backslash B_{z}$ in the interior of $T$. By definition the branch contains no cycles. Let us introduce the generalized Bruhat-Tits tree $F_{g}$. It consists of a finite connected graph $F_{g}^{R}$ with $g$ independent loops, which is called a reduced graph, the branches $B_{x}, x \in F_{g}^{R}$, and each vertex is connected by links with exactly $p+1$ nearest neighbours (for every link, two endpoints of which are identified with a vertex, we include the vertex itself twice into the number of its nearest neighbours). If the vertex $x \in F_{g}^{R}$ has only one nearest neighbour $y \in F_{g}^{R}, x \neq y$, then $p$ branches $B_{x}$ and the link $[x, y]$ form the branch $B_{y}$. Hence instead of the reduced graph $F_{g}^{R}$ we may consider the reduced graph $F_{g}^{\boldsymbol{R}} \backslash[x, y]$. From now on $F_{0}^{\boldsymbol{R}}$ is merely a single vertex and $p+1$ branches should be added to this vertex in order to construct the BruhatTits tree $F_{0}=T$, for $g>0$ each vertex $x \in F_{g}^{R}$ has $2 \leqq n(x) \leqq p+1$ nearest neighbours in $F_{g}^{R}$ and $b(x)=p+1-n(x)$ branches should be added to $x$ in order to construct the generalized Bruhat-Tits tree $F_{g}$. Due to [3-5] the Bruhat-Tits tree $T \equiv F_{0}$ may be interpreted as the coset space $P G L\left(2, \mathbb{Q}_{p}\right) / P G L\left(2, \mathbb{Z}_{p}\right)$, where $P G L(2, \mathbb{K})$ is the group of fractional linear transformations of the projective line $P^{1}(\mathbb{K})$ over a ring $\mathbb{K}$ (we deal with the field of $p$-adic numbers $\mathbb{Q}_{p}$ and with the ring of the $p$-adic integers $\left.\mathbb{Z}_{p}\right)$. The element of $G L\left(2, \mathbb{Q}_{p}\right)$ is called hyperbolic if it has eigenvalues which $p$-adic norms are different. A Schottky group $\Gamma_{g}$ is a free subgroup of $P G L\left(2, \mathbb{Q}_{p}\right)$ with $g$ generators, all non-unit elements of which are hyperbolic. Usually the generalized Bruhat-Tits tree $F_{g}$ may be interpreted as a coset space $T / \Gamma_{g}$, where $\Gamma_{g}$ is some Schottky group [4-6].

Since the configuration $\sigma$ takes the values \pm 1 the Ising model action may be rewritten in the form

$$
\beta \sum_{|x-y|=1} \sigma(x) \sigma(y)=\beta N_{1}-\beta / 2 \sum_{|x-y|=1}(\sigma(x)-\sigma(y))^{2},
$$


where $N_{1}$ is the total number of the links of the lattice. If $\sigma$ is allowed to take any real values we obtain the action for $\mathbb{R}$ Ising model

$$
S(\phi)=-\beta / 2 \sum_{|x-y|=1}(\phi(x)-\phi(y))^{2}
$$

by omitting the unessential term $\beta N_{1}$ and by changing $\sigma \rightarrow \phi$. This model is a lattice version of the free massless field. On the broad class of the lattices including the generalized Bruhat-Tits tree the correlation functions of the $\mathbb{R}$ Ising model with the free boundary conditions may be computed exactly [7]. By using the special average of these correlation functions the multiloop $p$-adic string amplitudes were calculated for the scattering of $N$ identical tachyons attached to the boundary of the generalized Bruhat-Tits tree $[8,9]$.

In this paper we calculate the partition function and the correlation functions of the Ising model on the generalized Bruhat-Tits tree with the free boundary conditions. We also compute the averages of these correlation functions which are analogous to the $p$-adic string amplitudes.

\section{Correlation Functions}

In order to find the correlation functions and partition function for the infinite generalized Bruhat-Tits tree $F_{g}$ we calculate them first on a finite subgraph $K \subset F_{g}$. A finite subgraph $K$ of the generalized Bruhat-Tits tree $F_{g}$ may be considered as a finite cell complex. It consists of zero- and one-dimensional cells: vertices and links. Every cell $\pm s_{i}^{q}$ is labelled by the integer $q=0,1$ (dimension) and by the number \pm 1 (orientation). The cells with the opposite orientation $s^{q}$ and $-s^{q}$ both belong to the lattice $K$. An integer-valued odd $\left(c^{q}\left(-s_{i}^{q}\right)=-c^{q}\left(s_{i}^{q}\right)\right)$ function $c^{q}$ on the $q$-dimensional cells is called a $q$-chain of the complex $K . c^{q}$ can be regarded as a formal sum $\sum m_{i} s_{i}^{q}$, where the integers $m_{i}=c^{q}\left(s_{i}^{q}\right)$. A set of $q$-chains is an Abelian group: $c^{q}+c^{\prime q}=\sum\left(m_{i}+m_{i}^{\prime}\right) s_{i}^{q}$. It is denoted by $C^{q}(K, \mathbb{Z})$. It is possible to introduce the inner product on $C^{q}(K, \mathbb{Z}):\left\langle c^{q}, c^{q}\right\rangle=\sum m_{i} m_{i}^{\prime}$. We define the boundary operator $\partial$ on $C^{q}(K, \mathbb{Z})$ by $\partial s^{0}=0$ and $\partial\left[x_{i}, x_{j}\right]=x_{j}-x_{i}$. By linearity it is easy to extend the boundary operator on $C^{q}(K, \mathbb{Z})$. We define the coboundary operator $\partial^{*}$ by the following relations $\partial^{*} c^{1}=0$ and $\left\langle\partial^{*} c^{0}, c^{\prime 1}\right\rangle=\left\langle c^{0}, \partial\left(c^{\prime 1}\right)\right\rangle$. A kernel $Z_{1}(K, \mathbb{Z})$ of a homomorphism $\partial: C^{1}(K, \mathbb{Z}) \rightarrow C^{0}(K, \mathbb{Z})$ is called a group of cycles of the complex $K$. The image $B_{0}(K, \mathbb{Z})$ of a homomorphism $\partial: C^{1}(K, \mathbb{Z}) \rightarrow C^{0}(K, \mathbb{Z})$ is called a group of boundaries of the complex $K$. The image $B^{1}(K, \mathbb{Z})$ of a homomorphism $\partial^{*}: C^{0}(K, \mathbb{Z}) \rightarrow C^{1}(K, \mathbb{Z})$ is called a group of coboundaries of the complex $K$.

A homomorphism of $C^{q}(K, \mathbb{Z})$ into the Abelian group $\mathbb{Z}_{2}=\{ \pm 1\}$ (Don't confuse with 2 -adic integers) is a $q$-chain of the complex $K$ with coefficients in $\mathbb{Z}_{2}$. A set of all these homomorphisms is an Abelian group which is denoted by $C^{q}\left(K, \mathbb{Z}_{2}\right)$. Each homomorphism $h^{q} \in C^{q}\left(K, \mathbb{Z}_{2}\right)$ is defined by its values on the $q$-chains $1 \cdot s_{i}^{q} \in C^{q}(K, \mathbb{Z})$ i.e. on the cells $s_{i}^{q}$. Thus $h^{q}$ is an $\mathbb{Z}_{2}$-valued function on the $q$-dimensional cells of $K$. On $C^{q}\left(K, \mathbb{Z}_{2}\right)$ we introduce the boundary and coboundary operators: $\partial h^{1}\left(c^{0}\right)=h^{1}\left(\partial^{*} c^{0}\right)$ and $\partial^{*} h^{0}\left(c^{1}\right)=h^{0}\left(\partial c^{1}\right)$. For example $\partial^{*} h^{0}([x, y])$ $=h^{0}(x) h^{0}(y)$, because of $\sigma^{-1}=\sigma$ for $\sigma= \pm 1$. The group of cycles $Z_{1}\left(K, \mathbb{Z}_{2}\right)$, the groups of boundaries $B_{0}\left(K, \mathbb{Z}_{2}\right)$ and coboundaries $B^{1}\left(K, \mathbb{Z}_{2}\right)$ are defined in an 
obvious way. The group $\mathbb{Z}_{2}$ is selfdual: if $\sigma_{1}, \sigma_{2}= \pm 1$, then

$$
\left\langle\sigma_{1} \mid \sigma_{2}\right\rangle=\sigma_{1}^{J\left(\sigma_{2}\right)}=\sigma_{2}^{J\left(\sigma_{1}\right)},
$$

where $J(\sigma)=1 / 2(1-\sigma)$. Analogously, if $c_{1}^{q}, c_{2}^{q} \in C^{q}\left(K, \mathbb{Z}_{2}\right)$ then

$$
\left\langle c_{1}^{q} \mid c_{2}^{q}\right\rangle=\prod_{s_{i}^{q} \in K}\left\langle c_{1}^{q}\left(s_{i}^{q}\right) \mid c_{2}^{q}\left(s_{i}^{q}\right)\right\rangle,
$$

where multiplication runs over all positively oriented links of the lattice $K$.

Let us consider the Ising model on the lattice $K$. A configuration is a chain $\sigma^{0} \in C^{0}\left(K, \mathbb{Z}_{2}\right)$, i.e. a function on the vertices of $K$ taking the values \pm 1 . The Ising model action may be rewritten as

$$
S\left(\sigma^{0}\right)=\beta \sum_{s_{i} \in K} \partial^{*} \sigma^{0}\left(s_{i}^{1}\right),
$$

where the summing runs over all positively oriented links of the lattice $K$. The partition function is

$$
Z_{k}=2^{-N_{0}} \sum_{\sigma^{0} \in C^{0}\left(K, \mathbb{Z}_{2}\right)} e^{S\left(\sigma^{0}\right)},
$$

where $N_{0}$ is the total number of the vertices of the lattice $K$. The correlation function has the form

$$
W_{K}\left(\chi^{0}\right)=Z_{K}^{-1} 2^{-N_{0}} \sum_{\sigma^{0} \in C^{0}\left(K, \mathbb{Z}_{2}\right)}\left\langle\chi^{0} \mid \sigma^{0}\right\rangle e^{S\left(\sigma^{0}\right)},
$$

where the chain $\chi^{0} \in C^{0}\left(K, \mathbb{Z}_{2}\right)$ takes the value -1 at the vertices $x_{1}, \ldots, x_{m}$ and takes the value 1 otherwise. Thus the definition (2.2) implies $\left\langle\chi^{0} \mid \sigma^{0}\right\rangle$ $=\sigma^{0}\left(x_{1}\right) \ldots \sigma^{0}\left(x_{m}\right)$.

By Lemma 1 of [10] the correlation function $W_{K}\left(\chi^{0}\right)$ isn't zero only for boundaries $\chi^{0}=\partial \chi^{1}$ and

$$
W_{K}\left(\partial \chi^{1}\right)=Z_{K}^{-1}\left|B^{1}\left(K, \mathbb{Z}_{2}\right)\right|^{-1} \sum_{\phi^{1} \in B^{1}\left(K, \mathbb{Z}_{2}\right)}\left\langle\chi^{1} \mid \phi^{1}\right\rangle \exp \left\{\beta \sum_{s_{i}^{i} \in K} \phi^{1}\left(s_{i}^{1}\right)\right\},
$$

where $\left|B^{1}\left(K, \mathbb{Z}_{2}\right)\right|$ is the order of the finite group of the coboundaries $B^{1}\left(K, \mathbb{Z}_{2}\right)$. By using the Fourier transformation on the group $B^{1}\left(K, \mathbb{Z}_{2}\right)$ we obtain due to Proposition 1 of [10]

$$
W_{K}\left(\partial \chi^{1}\right)=Z_{K}^{-1}(\operatorname{ch} \beta)^{N_{1}} \sum_{\xi^{1} \in Z_{1}\left(K, \mathbb{Z}_{2}\right)} \exp \left\{-2 \beta^{*} \sum_{s_{i}^{i} \in K} J\left(\chi^{1}\left(s_{i}^{1}\right) \xi^{1}\left(s_{i}^{1}\right)\right)\right\},
$$

where $J(\sigma)=1 / 2(1-\sigma)$ and the number $\beta^{*}$ is given by the following equation: $e^{-2 \beta^{*}}=\operatorname{th} \beta$.

Let $K$ be a finite connected subgraph of the generalized Bruhat-Tits tree $F_{g}$. Let $K$ contain also the reduced graph $F_{g}^{R}$. The cell complex $K$ is torsion free. Hence

$$
\begin{aligned}
\partial \chi^{1}\left(s_{k}^{0}\right) & =\exp \left\{i \pi\left(\partial c^{1}\right)\left(s_{k}^{0}\right)\right\}, \\
\xi^{1}\left(s_{k}^{1}\right) & =\exp \left\{i \pi \zeta^{1}\left(s_{k}^{1}\right)\right\},
\end{aligned}
$$

where $c^{1} \in C^{1}(K, \mathbb{Z})$ and $\zeta^{1} \in Z_{1}(K, \mathbb{Z})$. Since the graph $K$ is connected any chain $\partial c^{1}$ may be presented as $\partial\left(\sum_{1}^{N} m_{i} \kappa_{c, x_{i}}\right)$, where $\kappa_{c, x_{i}}$ is some path from the arbitrary fixed vertex $c \in F_{g}^{R}$ to the vertex $x_{i}$ and the integers $m_{i}$ satisfy the condition $\sum m_{i}=0$. (By 
definition the path $\kappa_{c, x_{i}}$ is the sum of the different links connecting two vertices $c$ and $x_{i}$.) In view of the first relation (2.8) it is possible to add to the chain $\partial c^{1}$ any chain $c^{0}=\sum n_{i} s_{i}^{0}$ with even integers $n_{i}$. Hence we may consider all $m_{i}=1$ and $N=\sum m_{i}=0(\bmod 2)$, i.e. $N$ is even. Since the graph $K$ contains the reduced graph $F_{g}^{R}$ and any branch has no loops each cycle $\zeta^{1} \in Z_{1}(K, \mathbb{Z})$ has the form $\zeta^{1}=\sum_{i=1}^{g} m_{i} z_{i}$ where the loops $z_{1}, \ldots, z_{g}$ form the basis of the group of cycles $Z_{1}\left(F_{g}^{R}, \mathbb{Z}\right)$. It is possible to choose the basis in this way thus $z_{k}\left(s_{i}^{1}\right)=0, \pm 1$ for any link $s_{i}^{1}$ and $k=1, \ldots, g$. In view of the second relation (2.8) we may consider all $m_{i}=0,1$. Thus the relation (2.8) may be rewritten as

$$
\begin{aligned}
\partial \chi^{1}\left(s_{k}^{0}\right) & =\partial\left(e^{i \pi \sum_{1}^{2 N} \kappa_{c, x_{j}}}\right)\left(s_{k}^{0}\right)=e^{i \pi \sum_{1}^{2 N} \delta_{x_{j}, s_{k}^{0}}} \\
\xi^{1}\left(s_{k}^{1}\right) & =\left(e^{i \pi \sum_{1}^{g} \varepsilon_{j} z_{j}}\right)\left(s_{k}^{1}\right),
\end{aligned}
$$

where the numbers $\varepsilon_{j}=0,1$.

It is easy to verify the following formula:

$$
1 / 2\left(1-e^{i \pi \sum_{1}^{n} \varepsilon_{k}}\right)=\sum_{q=1}^{n}(-2)^{q-1} \sum_{\substack{k_{1}<\ldots<<k_{q} \\ k_{j}=1}}^{n} \varepsilon_{k_{1}} \ldots \varepsilon_{k_{q}},
$$

where the numbers $\varepsilon_{k}=0,1$.

In view of the formula (2.10) the substitution of the relations (2.9) into Eq. (2.7) yields for the correlation function

$$
\begin{aligned}
& W_{K}\left(e^{i \sum_{j=1}^{2 N} \delta_{x_{j}}, \cdot}\right)=Z_{K}^{-1}(\operatorname{ch} \beta)^{N_{1}} \\
& \times \sum_{\varepsilon_{1}, \ldots, \varepsilon_{g}=0}^{1} \exp \left\{-2 \beta^{*}\left[\sum_{m=1}^{2 N}(-2)^{m-1} \sum_{k_{1}<\sum_{i}<k_{m}}^{2 N}\left\langle\kappa_{c, x_{k_{1}}}, \ldots, \kappa_{c, x_{k_{m}}}\right\rangle\right.\right. \\
& +\sum_{n=1}^{g}(-2)^{n-1} \sum_{l_{1}<\ldots i}^{g}<l_{n}\left\langle\varepsilon_{l_{1}} z_{l_{1}}, \ldots, \varepsilon_{l_{n}} z_{l_{n}}\right\rangle \\
& +\sum_{m=1}^{2 N} \sum_{n=1}^{g}(-2)^{m+n-1} \sum_{k_{1}<. . .1<k_{m}}^{2 N} \sum_{l_{1}<. . .1}^{g}<l_{n}\left\langle\kappa_{c, x_{k_{1}}}, \ldots, \kappa_{c, x_{k_{m}}},\right. \\
& \left.\left.\left.\varepsilon_{l_{1}} z_{l_{1}}, \ldots, \varepsilon_{l_{n}} z_{l_{n}}\right\rangle\right]\right\}
\end{aligned}
$$

and for the partition function

$$
Z_{K}=(\operatorname{ch} \beta)^{N_{1}} \sum_{\varepsilon_{1}, \ldots, \varepsilon_{g}=0}^{1} \exp \left\{-2 \beta^{*} \sum_{n=1}^{g}(-2)^{n-1} \sum_{l_{1}<\ldots i}^{g}\left\langle l_{i}\left\langle\varepsilon_{l_{1}} z_{l_{1}}, \ldots, \varepsilon_{l_{n}} z_{l_{n}}\right\rangle\right\}\right.
$$


Here for the chains $c_{1}^{1}, \ldots, c_{k}^{1} \in C^{1}(K, \mathbb{Z}), k=1,2, \ldots$, we introduced the product

$$
\left\langle c_{1}^{1}, \ldots, c_{k}^{1}\right\rangle=\sum_{s_{i}^{1} \in K}\left(c_{1}^{1}\left(s_{i}^{1}\right) \ldots c_{k}^{1}\left(s_{i}^{1}\right)\right) .
$$

For the Bruhat-Tits tree $T=F_{0}$ the formula (2.12) gives the partition function calculated in the paper [1].

If the vertices $x_{1}, \ldots, x_{2 N}$ are fixed, the limit $K \rightarrow F_{g}$ for the correlation function (2.11) is obtained by omitting the multiplier $(\operatorname{ch} \beta)^{N_{1}}$ in the formulas (2.11) and (2.12). We denote this limit by

$$
W\left(e^{i \pi \sum_{j=1}^{2 N} \delta_{x_{j}}, \cdot}\right)
$$

\section{Averages}

Our aim now will be to compute the correlation functions with the vertices attached to the boundary of the generalized Bruhat-Tits tree.

If a vertex $x_{i} \notin F_{g}^{R}$ then by the definition of the graph $F_{g}$ there exists the unique vertex $x^{R} \in F_{g}^{R}$ such that the path

$$
\kappa_{c, x}=\kappa_{c, x^{R}}+\kappa_{x^{R}, x},
$$

where the path $\kappa_{c, x^{R}}$ belongs to the reduced graph $F_{g}^{R}$ and the unique path $\kappa_{x^{R}, x}$ lies in the branch $B_{x^{R}}$. Any half-infinite path (without returns) in $B_{x^{R}}$ starting at an vertex $x^{R}$ we call a ray $x^{R} \rightarrow x$. The set of all rays will be called the boundary $\partial F_{g}$ of $F_{g}$. On $\partial F_{g}$ we introduce the basis of open sets $\partial B_{x}$, where $x \in F_{g} \backslash F_{g}^{R}$, and $\partial B_{x}$ consists of all rays having infinite intersection with the branch $B_{x}$. The measure $\mu_{0}$ on $\partial F_{g}$ is defined by the following relation:

$$
\mu_{0}\left(\partial B_{x}\right)=p^{-\left\langle\kappa_{x} R, x\right\rangle} .
$$

The relation

$$
\left|x^{R} \rightarrow x, y^{R} \rightarrow y\right|_{p}=p^{-\left\langle x^{R} \rightarrow x, y^{R} \rightarrow y\right\rangle}
$$

defines the distance on $\partial F_{g}$.

The reduced graph $F_{0}^{R}$ is merely a single vertex $c$. The boundary $\partial F_{0}$ can be naturally identified with $p$-adic projective line $P^{1}\left(\mathbb{Q}_{p}\right)[4,5]$ with the measure related to the Haar measure $d x$ on $\mathbb{Q}_{p}$ by the following relations:

$$
\begin{gathered}
d \mu_{0}(c \rightarrow x)=d x, \quad|x|_{p} \leqq 1, \\
d \mu_{0}(c \rightarrow x)=d x /|x|_{p}^{2}, \quad|x|_{p}>1,
\end{gathered}
$$

where $|\cdot|_{p}$ is the standard $p$-adic norm on $\mathbb{Q}_{p}$, and the distance (3.3) on $P^{1}\left(\mathbb{Q}_{p}\right)$ is defined by its restriction on $\mathbb{Q}_{p}$ :

$$
\begin{gathered}
|c \rightarrow x, c \rightarrow y|_{p}=|x-y|_{p}, \quad|x|_{p} \leqq 1, \quad|y|_{p} \leqq 1 ; \\
|c \rightarrow x, c \rightarrow y|_{p}=\left|x^{-1}-y^{-1}\right|_{p}, \quad|x|_{p}>1, \quad|y|_{p}>1 ; \\
|c \rightarrow x, c \rightarrow y|_{p}=1, \quad \text { otherwise. }
\end{gathered}
$$

We call the vertex $x^{R} \in F_{g}^{R}$ external if $x^{R}$ is the end of $b\left(x^{R}\right)>0$ branches in $F_{g}$. For $g>0$ by definition of the reduced graph an external vertex $x^{R} \in F_{g}^{R}$ defines $b\left(x^{R}\right)$ 
$\left(0<b\left(x^{R}\right) \leqq p-1\right)$ branches. Then the ray $x^{R} \rightarrow x$ starting at the external vertex $x^{R} \in F_{g}^{R}$ may be identified with the $p$-adic integer number $x \in \mathbb{Z}_{p}$ in the form

$$
x=a_{0}+a_{1} p+a_{2} p^{2}+\ldots,
$$

where $0 \leqq a_{0} \leqq b\left(x^{R}\right)-1$ and $0 \leqq a_{i} \leqq p-1$ for $i>0$. We denote the set of these numbers as $\mathbb{Z}_{p}\left[a_{0}<b\left(x^{R}\right)\right]$. Thus for $g>0$,

$$
\partial F_{g} \cong \bigcup_{\substack{x^{R} \in F_{g}^{R} \\ b\left(x^{R}\right)>0}} \mathbb{Z}_{p}\left[a_{0}<b\left(x^{R}\right)\right] .
$$

It is easy to verify that under this correspondence

$$
\begin{gathered}
\left|x^{R} \rightarrow x, y^{R} \rightarrow y\right|_{p}=\left(|x-y|_{p}\right)^{\delta_{x^{R}, y^{R}}}, \\
d \mu_{0}\left(x^{R} \rightarrow x\right)=d x .
\end{gathered}
$$

The distance between $k$ rays $x_{1}^{R} \rightarrow x_{1}, \ldots, x_{k}^{R} \rightarrow x_{k}$ is defined similarly to the definition (3.3)

$$
\left|x_{1}^{R} \rightarrow x_{1}, \ldots, x_{k}^{R} \rightarrow x_{k}\right|_{p}=p^{-\left\langle x_{1}^{R} \rightarrow x_{1}, \ldots, x_{k}^{R} \rightarrow x_{k}\right\rangle} .
$$

The definition of the generalized Bruhat-Tits tree $F_{g}$ and the relations (3.3), (3.10) imply

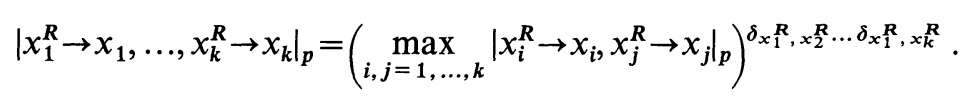

We call the boundary $\partial K$ of the graph $K$ the set of all vertices from $K \subset F_{g}$ which have among the nearest neighbours the vertices from $F_{g} \backslash K$. Let $f_{1}\left(x^{R} \rightarrow x\right), \ldots, f_{2 N}\left(x^{R} \rightarrow x\right)$ be the positive continuous functions summable with the measure $d \mu_{0}\left(x^{R} \rightarrow x\right)$ on $\partial F_{g}$ defined by the relation (3.2). We introduce the average

where

$$
A_{2 N}\left(f_{1}, \ldots, f_{2 N}\right)=\lim _{\substack{K_{j} \rightarrow F_{g} \\ j=1, \ldots, 2 N}} \sum_{\left.x_{j} \in \partial K_{j}\right\}} W\left(e^{i \pi} \sum_{j=1}^{2 N} \delta_{x_{j}}, \cdot\right) \prod_{j=1}^{2 N} \bar{f}_{j}\left(x_{j}\right),
$$

$$
\bar{f}_{j}\left(x_{j}\right)=\left(\mu_{0}\left(\partial B_{x_{j}}\right)\right)^{-1} \int_{\partial B_{x_{j}}} d \mu_{0}\left(x^{R} \rightarrow x\right) f_{j}\left(x^{R} \rightarrow x\right) .
$$

If all graphs $K_{j}=K$ and all functions $f_{j}\left(x^{R} \rightarrow x\right)=1$ the average $A_{2 N}(1, \ldots, 1)$ is the straightforward analogue of the $p$-adic string amplitude $[8,9]$.

We find the limit (3.12) for the special sequence of graphs $\left\{K_{1}\right\}$ such that

$$
\partial K_{l}=\left\{x \in F_{g} \mid\left\langle\kappa_{x^{R}, x}\right\rangle=l, x \in B_{x^{R}}, x^{R} \in F_{g}^{R}, b\left(x^{R}\right)>0\right\} .
$$

We suppose also that the supports of the functions $f_{i}\left(x^{R} \rightarrow x\right)$ and $f_{j}\left(x^{R} \rightarrow x\right)$ don't intersect for $i \neq j$.

For $g=0$ the relations (2.11), (2.12), (3.2) and (3.12), (3.13) imply

$$
\begin{aligned}
A_{2 N}\left(f_{1}, \ldots, f_{2 N}\right)= & \lim _{l \rightarrow \infty} \exp \left\{2 N l\left(\ln p-2 \beta^{*}\right)\right\} \\
& \times \sum_{\left\{x_{j} \in \partial K_{l}\right\}}\left(\prod_{j=1}^{2 N} \int_{\partial \boldsymbol{B}_{x_{j}}} d \mu_{0}(c \rightarrow x) f_{j}(c \rightarrow x)\right) \\
& \times \exp \left\{-2 \beta^{*} \sum_{m=2}^{2 N}(-2)^{m-1} \sum_{k_{1}<i_{i}<k_{m}}^{2 N}\left\langle\kappa_{c, x_{k_{1}}}, \ldots, \kappa_{\left.c, x_{k_{m}}\right\rangle}\right\} .\right.
\end{aligned}
$$


Since the supports of the functions $f_{i}\left(x^{R} \rightarrow x\right)$ and $f_{j}\left(x^{R} \rightarrow x\right)$ don't intersect for $i \neq j$ the last sum in (3.14) absolutely converges as $l \rightarrow \infty$ to

$$
\begin{aligned}
& \quad \int_{\mathbf{P}^{1}\left(\mathbb{Q}_{p}\right)^{\times 2 N}} f_{1}\left(x_{1}\right) d \mu_{0}\left(x_{1}\right) \ldots f_{2 N}\left(x_{2 N}\right) d \mu_{0}\left(x_{2 N}\right) \\
& \quad \times \prod_{m=2}^{2 N} \prod_{k_{1}<. .1<k_{m}}^{2 N}\left(\max _{i, j=1, \ldots, m}\left|x_{k_{i}}, x_{k_{j}}\right|_{p}\right)^{(-2)^{m-1} 2 \beta^{*}(\ln p)^{-1}} .
\end{aligned}
$$

Here we use the relations (3.3), (3.10), (3.11) and we denote by $d \mu_{0}(x)$ the measure given by the right-hand sides of the relations (3.4). The distance $|x, y|_{p}$ is given by the right-hand sides of the relations (3.5). We use also the correspondence $\partial F_{0} \cong P^{1}\left(\mathbb{Q}_{p}\right)[4,5]$ and replace the functions $f_{j}(c \rightarrow x)$ simply by $f_{j}(x)$.

It follows now from (3.14) that

If

$$
A_{2 N}\left(f_{1}, \ldots, f_{2 N}\right)=\left\{\begin{array}{ll}
0, & 2 \beta^{*}>\ln p \\
\infty, & 2 \beta^{*}<\ln p
\end{array} .\right.
$$

$$
2 \beta^{*}=\ln p
$$

inserting (3.15) into the right-hand side of (3.14) we obtain the non-trivial limit

$$
\begin{aligned}
& A_{2 N}\left(f_{1}, \ldots, f_{2 N}\right)=\int_{P^{1}\left(\mathbb{Q}_{p}\right)^{\times 2 N}} f_{1}\left(x_{1}\right) d \mu_{0}\left(x_{1}\right) \ldots f_{2 N}\left(x_{2 N}\right) d \mu_{0}\left(x_{2 N}\right) \\
& \quad \times \prod_{m=2}^{2 N} \prod_{k_{1}<\ldots<k_{m}}^{2 N}\left(\max _{i, j=1, \ldots, m}\left|x_{k_{i}}, x_{k_{j}}\right|_{p}\right)^{(-2)^{m-1}} .
\end{aligned}
$$

Here we considered the simplest case when the supports of the different functions $f_{i}(x)$ on $P^{1}\left(\mathbb{Q}_{p}\right)$ don't intersect. In order to extend the formula (3.18) to the general case it is necessary to study the convergence of the integral (3.18).

Let us consider the generalized Bruhat-Tits tree $F_{g}$ with $g>0$. By using the relations (2.11), (2.12), the decomposition (3.1) and the formulas (3.7)-(3.11) we obtain the relation (3.16). If $\beta^{*}$ satisfies the condition (3.17) we have the non-trivial limit

$$
\begin{aligned}
& A_{2 N}\left(f_{1}, \ldots, f_{2 N}\right)=\sum_{\substack{\left\{x_{i}^{R}\right\} \in F_{F}^{R} \\
b\left(x^{R}\right)>0}} W\left(e^{i \pi_{j=1}^{2 N} \delta_{j}^{R}, \cdot}\right) \\
& \times \int_{\mathbb{Z}_{p}\left[a_{0}<b\left(x_{1}^{R}\right)\right]} f_{1}\left(x_{1}^{R} \rightarrow x_{1}\right) d x_{1} \ldots \int_{\mathbb{Z}_{p}\left[a_{0}<b\left(x_{2 N}^{R}\right)\right]} f_{2 N}\left(x_{2 N}^{R} \rightarrow x_{2 N}\right) d x_{2 N} \\
& \times \prod_{m=2}^{2 N} \prod_{k_{1}<. .1<k_{m}}^{2 N}\left(\max _{i, j=1, \ldots, m}\left|x_{k_{i}}-x_{k_{j}}\right|_{p}\right)^{(-2)^{m-1} \delta_{x k_{1}}^{R}, x_{k_{2}}^{R} \ldots \delta_{x k_{1}}^{R}, x_{k_{m}}^{R}},
\end{aligned}
$$

where the correlation function

$$
W\left(e^{i \pi \sum_{j=1}^{2 N} \delta_{x_{j}^{R}}^{R}, \cdot}\right)
$$

is given by the equations (2.11), (2.12) with the omitted multiplier $(\operatorname{ch} \beta)^{N_{1}}$. The proof of the formula (3.19) is exactly analogous to the case $g=0$. To extend the formula (3.19) to the case when the supports of the functions $f_{1}\left(x^{R} \rightarrow x\right)$ intersect one needs to study the convergence of the integral (3.19). 


\section{References}

1. Eggarter, T.P.: Cayley trees, the Ising problem, and the thermodynamic limit. Phys. Rev. B 9, 2989-2992 (1974)

2. Müller-Hartmann, E., Zittartz, J.: New type of phase transition. Phys. Rev. Lett. 33, 893-897 (1974)

3. Bruhat, F., Tits, J.: Groupes reductifs sur un corps local. I. Données radicielles valuées. Publ. Math. IHES 41, 5-251 (1972)

4. Serre, J.P.: Trees. Berlin, Heidelberg, New York: Springer 1980

5. Manin, Yu.I.: p-Adic automorphic functions. Sovr. Probl. Mat. 3, 5-92. Moscow: VINITI 1974 (in Russian)

6. Gerritzen, L., van der Put, M.: Schottky groups and Mumford curves. Lecture Notes in Math. vol. 817. Berlin, Heidelberg, New York: Springer 1980

7. Zinoviev, Yu.M.: Lattice $\mathbb{R}$-gauge theories. Theor. Math. Phys. 49, No. 2 (1981)

8. Zabrodin, A.V.: Non-archimedean strings and Bruhat-Tits trees. Commun. Math. Phys. 123, 463-483 (1989)

9. Chekhov, L.O., Mironov, A.D., Zabrodin, A.V.: Multiloop calculations in p-adic string theory and Bruhat-Tits trees. Commun. Math. Phys. 125, 675-711 (1989)

10. Zinoviev, Yu.M.: Duality in the Abelian lattice theories. Theor. Math. Phys. 43, 481-490 (1980)

Communicated by Ya. G. Sinai

Received November 11, 1989 